\title{
Beam dynamics issues for the two-frequency crab cavity short pulse scheme
}

\author{
Xiaobiao Huang $\odot^{*}$ Bob Hettel, Tom Rabedeau, James Safranek, Jim Sebek, \\ Kai Tian, and Kent P. Wootton \\ SLAC National Accelerator Laboratory, Menlo Park, California 94025, USA \\ A. Zholents \\ Argonne National Laboratory, Lemont, Illinois 60439, USA
}

(Received 5 June 2019; published 24 September 2019)

\begin{abstract}
We report the study of various beam dynamics issues arising from the scheme of using two-frequency crab cavities to generate short pulse $\mathrm{x}$-rays for timed-resolved experiments in a storage ring while simultaneously supplying high brightness beams to other users. These issues include the prediction of the short pulse performance, the choice of system parameters, the impact of the crab cavities on injection efficiency and beam lifetime of the camshaft bunch, and the impact of crab cavities on the regular beam. The collective effect requirements and the separation of short pulses and regular beams are also discussed. With this scheme applied to the SPEAR3 ring, the short pulse flux would be equivalent to a $0.5 \mathrm{nC}$ bunch with a duration of $3 \mathrm{ps}$ (full width half maximum, fwhm), or a $2 \mathrm{nC}$ bunch with a duration of $7 \mathrm{ps}$ (fwhm).
\end{abstract}

DOI: 10.1103/PhysRevAccelBeams.22.090703

\section{INTRODUCTION}

Short x-ray pulses with durations of 1-10 ps fwhm from storage ring light sources would enable the study of many dynamic processes in materials. Such short pulse capabilities would complement the extremely bright and short pulses from x-ray FELs in many aspects, such as preservation of samples, linear response probing, low cost, and high availability.

The Stanford Synchrotron Radiation Lightsource (SSRL) user community has identified that a timing mode would be highly desirable if, (1) it is compatible with and has minimal impact to the regular $500 \mathrm{~mA}$, high brightness mode; (2) its pulse duration is $10 \mathrm{ps}$ fwhm or below; (3) short pulses can be isolated from the regular pulses; (4) the monochromatized photon flux $\left(\sim 10^{-4}\right.$ bandwidth $)$ is of the order of $10^{12} \mathrm{ph} / \mathrm{s}$ from the short pulses.

After an investigation of a number of possible short pulse approaches, including, low alpha operation [1], resonant crabbing [2,3], short pulse injection from the LCLS-II superconducting (SC) linac [4,5], longitudinal bunch compression with two-frequency SC harmonic rf cavities [6,7], transverse bunch crabbing and cancellation within one turn [8,9], and transverse bunch crabbing using two-frequency crab cavities (2FCC) [10], we came to the conclusion that

\footnotetext{
*xiahuang@slac.stanford.edu

Published by the American Physical Society under the terms of the Creative Commons Attribution 4.0 International license. Further distribution of this work must maintain attribution to the author(s) and the published article's title, journal citation, and DOI.
}

the $2 \mathrm{FCC}$ scheme provides the optimal solution for the SSRL short pulse upgrade in terms of short pulse performance, technical difficulty, and cost effectiveness.

In the 2FCC scheme, crab cavities of two frequencies, one a harmonic of the fundamental radio frequency (rf), the other of a half harmonic, are used to provide an arrival timedependent vertical kick to selected bunch(es), which puts the bunches in an equilibrium distribution with a tilt across the vertical-longitudinal planes. The two frequencies are chosen so that the crabbing kicks cancel for alternate rf buckets in which the regular beam is stored.

Compared to the crab-and-cancel [8] scheme previously pursued by the APS-SPX (short pulse X-ray) project [9], the new crab cavity scheme has several advantages: short pulses are available all around the ring; no strict betatron phase advance between cells is required for the lattice; crab cavities occupy only one straight section, which saves space and cost; less total deflecting voltage is required since crab cavities of both frequencies contribute to tilting the camshaft bunch; crab cavities can provide separation between the short pulses and the regular beam; and beamlines can easily switch between short pulse mode and regular beam mode.

At SSRL, we have studied the possibility to apply the 2FCC scheme to the SPEAR3 storage ring. We studied a variety of beam dynamics issues for the scheme that could have an impact on the short pulse and/or regular beam performance. The results of the studies are reported in this paper. The system layout of the 2FCC scheme for the SPEAR3 case is described in Sec. II. The prediction of the short pulse performance is presented in Sec. III. In Sec. IV 
TABLE I. The 2FCC lattice parameters for SPEAR3.

\begin{tabular}{lc}
\hline \hline Parameters (unit) & Values \\
\hline Beam Energy $(\mathrm{GeV})$ & 3 \\
Circumference $(\mathrm{m})$ & 234.1 \\
Betatron tunes $\left(\nu_{x}, \nu_{y}\right)$ & $(15.10,6.32)$ \\
Camshaft current $(\mathrm{mA})$ & 20 \\
Main rf frequency $(\mathrm{MHz})$ & 476.30 \\
Crab cavity frequency 1 $(\mathrm{GHz})$ & 2.8578 \\
Crab cavity frequency 2 $(\mathrm{GHz})$ & 3.0960 \\
Horizontal emittance $(\mathrm{nm} \cdot \mathrm{rad})$ & 7 \\
Rms bunch length @ $I_{b}=0(\mathrm{ps})$, & 19 \\
Rms bunch length @ $I_{b}=20 \mathrm{~mA}(\mathrm{ps})$, & 30 \\
Rms momentum deviation, $\sigma_{\delta}$ & 0.001 \\
Vertical emittance w/o crab cavities $(\mathrm{pm} \cdot \mathrm{rad})$ & 5 \\
Vertical beta at crab cavities, $\beta_{2}(\mathrm{~m})$ & 2.5 \\
Vertical damping time, $\tau_{y}(\mathrm{~ms})$ & 5.3 \\
Longitudinal damping time, $\tau_{s}(\mathrm{~ms})$ & 4.1 \\
\hline \hline
\end{tabular}

we discuss single particle dynamics issues related to the camshaft bunch, such as injection into the tilted bunch and beam lifetime. In Sec. V the impact of the crab cavities on the regular beam, including the effects of rf noise and the effects of phase shifts due to transient beam loading, is discussed. Section VI reports considerations of collective effects. Section VII discusses two practical aspects, the selection of the crab cavity frequencies and the separation of long and short pulses. A summary is given in Sec. VIII.

\section{SYSTEM LAYOUT OF THE TWO-FREQUENCY CRAB CAVITY SCHEME FOR SPEAR3}

SPEAR3 is a third generation light source with an electron beam energy of $3 \mathrm{GeV}$. The horizontal emtitance is $10 \mathrm{~nm}$ and the ring circumference is $234 \mathrm{~m}$. We are currently in the process of upgrading the septum magnet which will allow the operation of a new lattice with emittance at $7 \mathrm{~nm}$ [11]. Table I gives a list of the relevant storage ring and 2FCC parameters.

The two crab cavity frequencies are chosen to be $6 \times$ and $6.5 \times$ of the $476.3 \mathrm{MHz}$ fundamental rf, with $f_{1}=$ $2.858 \mathrm{GHz}$ and $f_{2}=3.096 \mathrm{GHz}$, respectively. The nominal deflecting voltage for the 6th harmonic crab cavity is $V_{1}=1.0 \mathrm{MV}$, while the deflecting voltage for the second frequency is $V_{2} \approx 0.93 V_{1}$ as required to minimize the distortion to the regular beam [10]. The crab cavities are operated at zero-crossing so that their main effects on the beam are to tilt the bunches vertically through the $z$-dependent vertical kicks. For half of the buckets, the deflecting slopes of the two crab cavity systems will add up. Any bunch in these buckets will acquire a steady-state, vertical-longitudinal tilted equilibrium distribution. For the other half of the buckets, the deflecting slopes of the two systems cancel.

The crab cavities will be placed in a $4.5 \mathrm{~m}$ straight section. Having the proper frequencies and deflecting voltages for the two crab cavities systems alone cannot ensure the complete cancellation of their tilting effects as the cavities cannot physically occupy the same space. As the beam travels from one crab cavity to the next, the angular kicks by the first crab cavity have translated to position shifts, which cannot be removed by the kicks of the second crab cavity. For complete cancellation, in the straight section that houses the crab cavities, the conditions

$\int_{0}^{L} g\left(s^{\prime}\right) d s^{\prime}=0, \quad$ and $\quad \int_{0}^{L} d s \int_{s}^{L} g\left(s^{\prime}\right) d s^{\prime}=0$,

need to be satisfied, where $g=\frac{d y^{\prime}}{d t}$ is the deflecting slope. It is easy to show that if the crab cavities of the two frequencies are arranged symmetrically about the center of the straight section and that the first condition is satisfied, the second condition will also be satisfied. One such arrangement would consist of four crab cavities placed in the order of $\left(\frac{V_{1}}{2}, f_{1}\right),\left(\frac{V_{2}}{2}, f_{2}\right),\left(\frac{V_{2}}{2}, f_{2}\right)$, and $\left(\frac{V_{1}}{2}, f_{1}\right)$.

With the crab cavities of the two frequencies properly arranged, every other rf bucket will be minimally impacted by the crab cavities. These buckets will be filled to a high total current for regular photon beam users. Beam in the other buckets will be tilted. For the timing mode, only one or at most a few tilted bunches are needed. Each timing bunch is separated from the other beam bunches with large gaps and is called a camshaft bunch. A possible fill pattern for the SPEAR3 2FCC operation with one camshaft bunch is illustrated in Fig. 1, where the blue ellipses represent buckets with filled bunches and empty ellipses represent empty buckets.

Generally the camshaft bunch will have a tilted distribution at the radiation source points (i.e., undulators) in both the $y-z$ and $y^{\prime}-z$ phase planes. The radiation by such an electron bunch will also be tilted. A vertical slit is placed in the photon beamline to select short pulses out of the photon beams. The optics between the source point and the pulse selection slit can be a simple drift, or an imaging system. The two types of optics are illustrated in Fig. 2. For a given

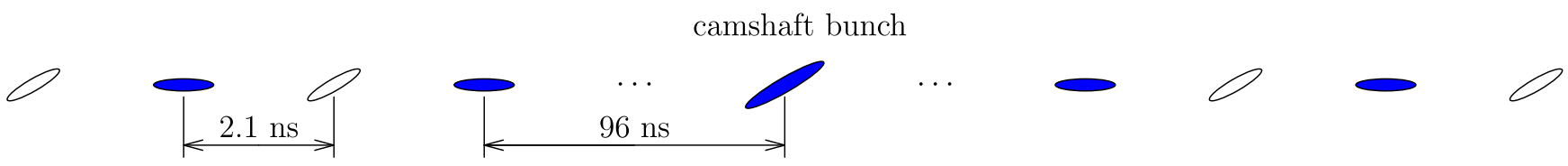

FIG. 1. Bunch pattern for the SPEAR3 2FCC mode. The camshaft bunch current is $20 \mathrm{~mA}$ in a single bunch. The total regular beam current is $480 \mathrm{~mA}$, equally distributed in 138 nontilted bunches (blue). 


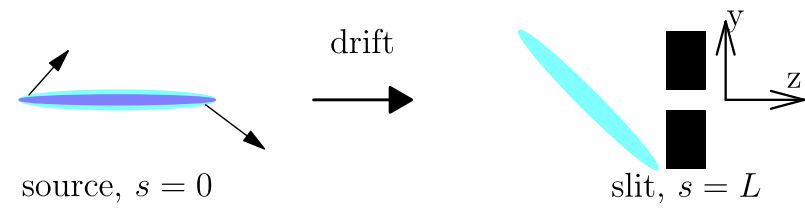

(a) drift optics
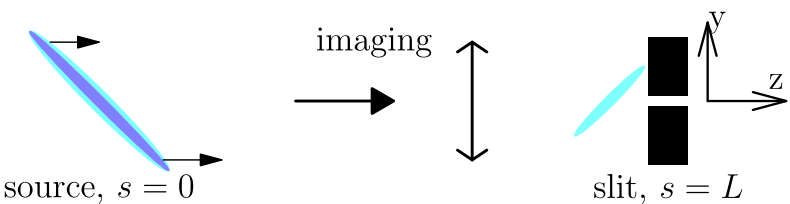

(b) imaging optics

FIG. 2. Illustration of two photon beam optics between source points and pulse selection slits. (a) $y^{\prime}-z$ tilt at the source point becomes $y-z$ tilt at the short pulse selection slit; (b) the photon beam with a $y-z$ slope at the source point is imaged to the slit.

beamline, drift or imaging optics may be chosen based on the photon beam distribution at the source point, distance between the source point and the slit, and potentially other considerations.

\section{SHORT PULSE PERFORMANCE}

\section{A. Equilibrium distribution of the tilted bunch}

Crab cavities of both frequencies work at the zerocrossing of the rf waveforms at the bucket centers. For the tilted bunch, the kick vs time slopes for both frequencies have the same sign and their effects on the beam add up. The combined time dependent kick couples the particle motion in the vertical plane and the longitudinal plane. This coupling causes a tilt of the equilibrium distribution across the phase space planes $\left(y, y^{\prime}\right)$ and $(z, \delta)$. The tilt angles at each location are related to the off-diagonal elements of the local decoupling matrix [12]. For example,

$$
\begin{gathered}
\frac{d y}{d z}=C_{11} \approx \epsilon \frac{\sqrt{\beta_{1} \beta_{2}}}{2 \sin \pi \nu_{y}} \cos \left(\pi \nu_{y}-\Psi_{12}\right), \\
\frac{d y^{\prime}}{d z}=C_{21} \\
\approx \epsilon \frac{\sqrt{\beta_{2} / \beta_{1}}}{2 \sin \pi \nu_{y}}\left[\sin \left(\pi \nu_{y}-\Psi_{12}\right)-\alpha_{1} \cos \left(\pi \nu_{y}-\Psi_{12}\right)\right],
\end{gathered}
$$

where $\beta_{2}$ and $\beta_{1}$ are vertical beta functions at the crab cavity location (point 2) and the observation point (point 1), respectively, $\nu_{y}$ is the vertical tune, $\alpha_{1}$ is the CourantSnyder alpha parameter at the observation point, $\Psi_{12}$ is the vertical phase advance from the crab cavity to the observation point, and $\epsilon$ is the crab cavity coupling strength.
For the 2FCC scheme, the deflecting slope consists of contributions from both frequencies,

$$
\epsilon=\frac{e\left(k_{1} V_{1}+k_{2} V_{2}\right)}{E},
$$

where $k_{1,2}=2 \pi f_{1,2} / c$ are the angular wave numbers and $V_{1,2}$ the deflecting voltages for the two frequencies, respectively. The approximation in Eqs. (2)-(3) was obtained by ignoring the slow synchrotron motion.

Reference [12] studied the vertical eigenemittance increases due to the tilted distributions in the $y-\delta$, and $y^{\prime}-\delta$ planes, as photon emission excites beam motion around these tilted distributions. The increase in vertical emittance was found to be

$$
\epsilon_{y}=C_{q} \gamma^{2} \frac{\epsilon^{2} C^{2} \alpha_{c}^{2} \beta_{2}}{12 J_{y} \rho} \frac{2+\cos 2 \pi \nu_{y}}{\cos 2 \pi \nu_{s}-\cos 2 \pi \nu_{y}},
$$

where $C_{q}=3.83 \times 10^{-13} \mathrm{~m}, \gamma$ is the Lorentz factor, $J_{y}=1$ is the vertical damping partition number, $\rho$ is the bending radius, $C$ is the ring circumference, $\alpha_{c}$ is the momentum compaction factor, and $\nu_{s}$ is the synchrotron tune. The tilt induced vertical emittance increase has a strong dependence on the momentum compaction factor and the vertical tune. Increasing $\nu_{y}$ toward the half integer reduces the emittance increase.

\section{B. Calculation of short pulse performance}

The short pulse flux selected by a certain slit aperture is closely related to the short pulse duration. When the slit is opened more, flux will increase; but the pulse duration will also increase. The performance of the short pulses may be characterized by the percentage of the short pulse flux over the total flux of the electron bunch vs the pulse duration.

If the photon beam distribution in the $y-z$ plane at the slit, $\rho(y, z)$, is known, the short pulse line density for a given slit aperture, $-a<y<a$ can be calculated with

$$
\lambda(z ; a)=\int_{-a}^{a} \rho(y, z) d y,
$$

from which the percentage of flux, $F(a)$, and the short pulse duration $\sigma_{z}(a)$ can be obtained, using

$$
F(a)=\int_{-\infty}^{\infty} \lambda(z ; a) d z, \quad \sigma_{z}^{2}(a)=\frac{1}{F(a)} \int_{-\infty}^{\infty} z^{2} \lambda(z ; a) d z .
$$

The minimum pulse duration can be calculated by taking the limit $a \rightarrow 0$ for $\sigma_{z}(a)$. For a Gaussian distribution

$$
\rho(y, z)=\frac{1}{2 \pi \epsilon_{y z}} \exp \left(-\frac{\sigma_{z}^{2} y^{2}-2 \sigma_{y z} y z+\sigma_{y}^{2} z^{2}}{2 \epsilon_{y z}^{2}}\right),
$$


with $\epsilon_{y z} \equiv \sqrt{\sigma_{z}^{2} \sigma_{y}^{2}-\sigma_{y z}^{2}}$, the minimum duration is given by

$$
\sigma_{z, \min }=\frac{\epsilon_{y z}}{\sigma_{y}} .
$$

The photon beam distribution at the slit is related to the distribution at the source point through the transfer matrix of the beam line optics between the two points. For example, if the beam line consists of only a drift space of length $L$, the transfer matrix, $\mathbf{M}$, for the coordinate vector $\mathbf{X}=\left(y, y^{\prime}, z, \delta\right)^{T}$ differs from the 4-by-4 identity matrix by only one element, namely, $M_{12}=L$. The second order moment matrix of the beam, $\boldsymbol{\Sigma}=\left\langle\mathbf{X}^{T} \mathbf{X}\right\rangle$, at the two locations are related through

$$
\boldsymbol{\Sigma}_{1}=\mathbf{M} \boldsymbol{\Sigma}_{0} \mathbf{M}^{T},
$$

where subscripts 0 and 1 indicate the source point and the slit, respectively, $\langle\cdot\rangle$ represents taking the ensemble average over the particles in the beam, and $\mathbf{M}^{T}$ is the transpose of $\mathbf{M}$.

The photon beam distribution at the source point can be obtained by the convolution of the electron beam distribution and the radiation distribution of a single electron at the source point. For an undulator source, the single electron radiation is often approximated with a Gaussian distribution with $\sigma_{\theta} \sigma_{r}=\lambda / 4 \pi$ and $\sigma_{\theta}=\sqrt{\lambda / 2 L_{\text {und }}}$, where $\lambda$ is the radiation wavelength, $L_{\text {und }}$ is the undulator length, $\sigma_{\theta}$ is the rms radiation angle, and $\sigma_{r}$ is the rms radius of the radiation at the source point.

Knowing the electron beam distribution at the source point, the radiation characteristics, and the optics between the source point and the slit, we can compute the photon beam distribution at the slit and the short pulse performance. Because of the finite momentum spread, the tilts of $y$ and $y^{\prime}$ with respect to $\delta$ also has an impact. From the equilibrium distribution of the electron beam found in previous studies. [12], its contribution to the minimum pulse duration is estimated to be only a small fraction of the total duration, with the ratio on the order of $\frac{\alpha_{c} C \sigma_{\delta}}{2 \sigma_{z}}=\pi \nu_{s}$. For the SPEAR3 case, the correction to pulse duration due to the tilts with momentum is only $\sim 3 \%$ as $\nu_{s} \approx 0.01$. Dropping the momentum spread terms and assuming $\alpha_{1}=0$, the minimum pulse duration for the case of a simple drift optics between the source point and the slit is found to be

$$
\begin{aligned}
\sigma_{z, \min } & =\frac{1}{C_{11}+C_{21} L} \sqrt{\left(\beta_{y}+\frac{L^{2}}{\beta_{y}}\right) \epsilon_{y}+\left(L^{2}+\beta_{\mathrm{ph}}^{2}\right) \sigma_{\theta}^{2}}, \\
& \approx \frac{1}{C_{11} / L+C_{21}} \sqrt{\frac{\epsilon_{y}}{\beta_{y}}+\sigma_{\theta}^{2}},
\end{aligned}
$$

where $C_{11,21}$ are elements of the coupling matrix [12], $\beta_{y}$ is the beta function of the electron beam at the source point, $\epsilon_{y}$ is the vertical emittance, $\beta_{\mathrm{ph}} \equiv \sigma_{r} / \sigma_{\theta}=L_{\text {und }} / 2 \pi$, and the approximation is valid under the assumptions $\beta_{p h} \ll L$ and $\beta_{y} \ll L$, which are valid in the case under consideration.

For the best short pulse performance with a drift optics, a small spread in the vertical angle coordinate for electrons and a large $y^{\prime}-z$ tilt at the source point are preferred. Inserting Eqs. (2)-(3) into Eq. (12) and assuming the appropriate phase advance $\Psi_{1,2}$ resulting in minimal pulse duration, we obtain

$$
\sigma_{z, \min }=\frac{2 \sin \pi \nu_{y}}{\epsilon \sqrt{1+\beta_{y}^{2} / L^{2}}} \sqrt{\frac{\epsilon_{y}}{\beta_{2}}+\frac{\beta_{y}}{\beta_{2}} \sigma_{\theta}^{2}},
$$

which can be used for a quick estimate of the minimum pulse duration.

In the case with an imaging system between the source point and the slit, the transfer matrix represents a scaling of the $y$ and $y^{\prime}$ coordinates, with scaling factors $M_{11}=r$ and $M_{22}=1 / r$, respectively, where $r$ is the magnification factor of the imaging optics. The minimum short pulse duration can be similarly calculated and is found to be

$$
\sigma_{z, \min }=\frac{1}{C_{11}} \sqrt{\beta_{y} \epsilon_{y}+\sigma_{r}^{2}} .
$$

A large magnitude of $C_{11}$, the $y-z$ slope, is critical for obtaining short pulse duration for the imaging optics case, while a large $C_{21}$ is critical for the drift optics case. Under favorable phase advance, the minimum pulse duration can be estimated with

$$
\sigma_{z, \min }=\frac{2 \sin \pi \nu_{y}}{\epsilon} \sqrt{\frac{\epsilon_{y}}{\beta_{2}}+\frac{\sigma_{r}^{2}}{\beta_{2} \beta_{y}}} .
$$

From Eqs. (2)-(3) we see that $C_{11}$ and $C_{21}$ oscillations with respect to the betatron phase advance are out of phase by $\pi / 2$ (noting that typically $\alpha_{1}=0$ at the insertion devices). Therefore, a beamline that is not suitable for short pulse selection with the drift optics would usually be ideal for the imaging optics, and vice versa. In the above we considered two special beamline optics between the source point and the short pulse selection slit—a drift space or an imaging system. It is worth pointing out that with a given tilted $\left(y, y^{\prime}\right)-z$ distribution, it may be possible to design the beamline optics, combining drifts and focusing elements, such that the transfer matrix between the source point and the slit maximizes the $y-z$ tilt of the photon beam at the slit, in which case the short pulse performance is optimized.

Using Eq. (5), the vertical emittance due to crab cavities is calculated to be $69 \mathrm{pm}$. The total vertical emittance, including the contribution from $x-y$ coupling, 
can be assumed to be $80 \mathrm{pm}$. The minimum pulse length for drift optics is estimated to be $3.1 \mathrm{ps}$ (fwhm) at standard straight section beamlines (where $\beta_{y}=5 \mathrm{~m}$ ), $2.3 \mathrm{ps}$ (fwhm) at chicane straight sections (where $\beta_{y}=1.6 \mathrm{~m}$ ), and $2.5 \mathrm{ps}$ (fwhm) at matching straight sections, assuming $\sigma_{\theta}=6.4 \mu \mathrm{rad}$ and $L=10 \mathrm{~m}$. The minimum pulse length for imaging optics is $1.9 \mathrm{ps}$ (fwhm) for all three types of locations, as the electron beam emittance is the dominant term in this case (assuming $\sigma_{r}=1.9 \mu \mathrm{m}$ ).

\section{Simulation for performance prediction}

Particle tracking has been conducted to obtain the equivalent electron beam distribution and in turn the short pulse performance for the SPEAR3 2FCC scheme. Relevant SPEAR3 parameters for the future 2FCC scheme can be found in Table I.

The tracking code Accelerator Toolbox (AT) [13] was used in the tracking study. We added new functions to AT to simulate crab cavities and quantum excitation in dipole magnets. The AT tracking results with the new functions were benchmarked to the tracking code Elegant [14] and good agreement was found between the two codes. In the tracking study, 10000 particles were launched from the

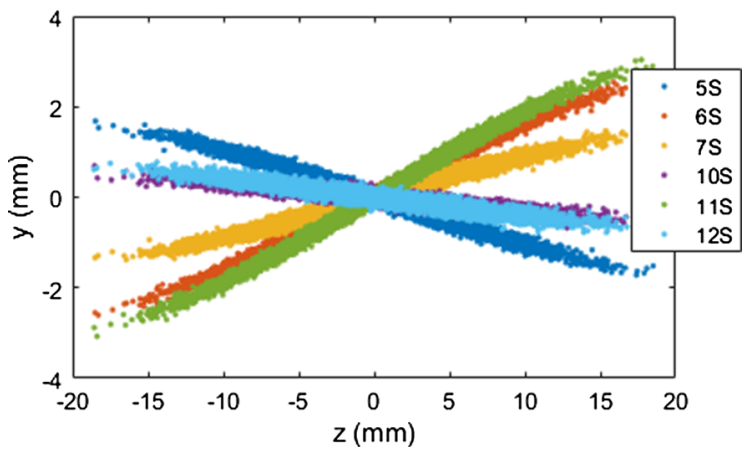

(a)

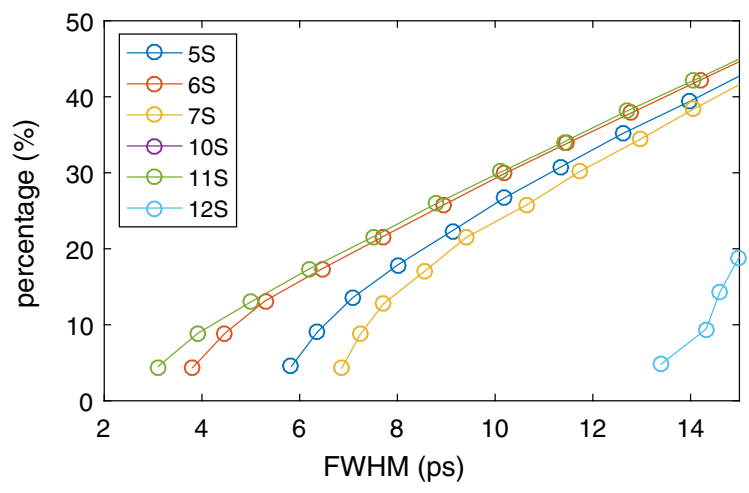

(c) phase space origin and tracked for 30000 turns, corresponding to 4.4 vertical damping times. The particles settle to the equilibrium distribution as the radiation damping is balanced by quantum excitation.

The equilibrium electron distribution was transported to the source points of beamlines of interest, and then random deviations were added to the $y$ and $y^{\prime}$ coordinates of all particles to generate the photon distribution. The position and angle deviations were drawn from Gaussian distributions with $\sigma_{r}$ and $\sigma_{\theta}$ at appropriate values for the beamlines at $8 \mathrm{keV}$ photon beam energy. For example, we used the values of $\sigma_{\theta}=6.4 \mu \mathrm{rad}$ for BL-15 and BL 12-1, and $\sigma_{\theta}=7.2 \mu \mathrm{rad}$ for a proposed new in-vacuum undulator in 10S straight section.

The photon beam is then transported downstream in the photon beamline to the short pulse selection slit. For the drift optics case, the slit is assumed to be located at $10 \mathrm{~m}$ from the source point. The slit with various apertures is then applied to the photon beam, and the flux percentage and the short pulse duration are evaluated for the selected pulse. In Fig. 3 we show the photon beam distribution at the slit and the short pulse flux percentage vs short pulse duration curves for existing and potential beamlines, assuming the

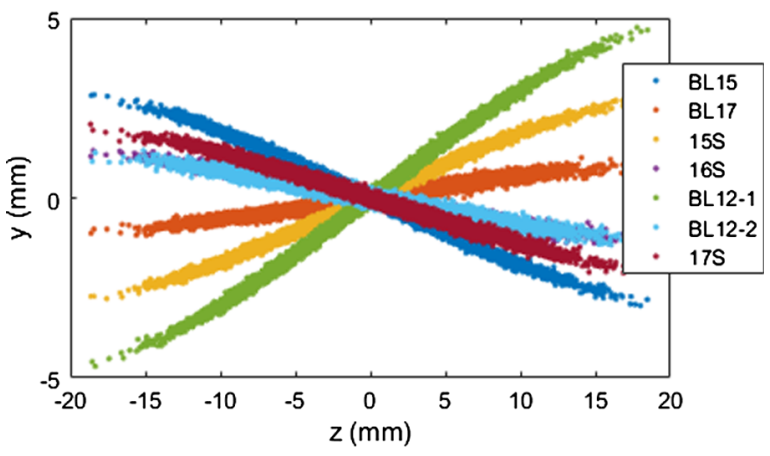

(b)

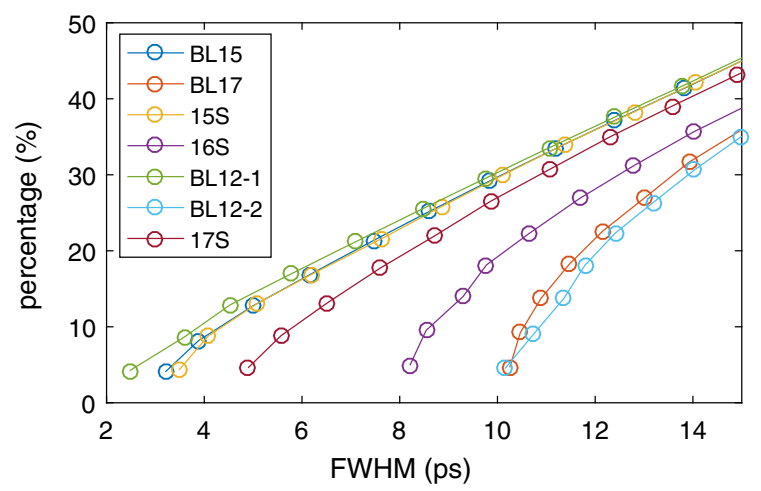

(d)

FIG. 3. (a-b) Photon beam distribution at the slit for the SPEAR3 case with simple drift between the source points and the slits. (c-d) The flux percentage vs short pulse duration, not considering bunch lengthening. The crab cavities are located in the 1S straight section and deflecting voltage for the first frequency is $V_{1}=1 \mathrm{MV}$. Beside the existing undulator beamlines, other straight sections that could be upgraded are also shown (as indicated by $5 \mathrm{~S}, 6 \mathrm{~S}$, etc.), using BL15 photon parameters. 
TABLE II. Short pulse duration (fwhm) for the SPEAR3 2FCC scheme for various flux percentages. Percentages in parentheses include bunch lengthening for a $20 \mathrm{~mA}$ bunch charge. Crab cavities are located in $1 \mathrm{~S}$, with $V_{1}=1 \mathrm{MV}$. Pulse duration degradation for the 10S-BL (w/imaging) is about $\sim 30 \%$ (not included).

\begin{tabular}{lccccc}
\hline \hline & & \multicolumn{4}{c}{ Short pulse duration (fwhm, ps) } \\
\cline { 3 - 6 } & Optics & $6 \%$ & $10 \%$ & $15 \%$ & $20 \%$ \\
Beam line & type & $(3.6 \%)$ & $(6.3 \%)$ & $(9.4 \%)$ & $(12.6 \%)$ \\
\hline BL15 & Drift & 3.5 & 4.3 & 5.6 & 7.1 \\
BL12-1 & Drift & 2.9 & 3.9 & 5.2 & 6.7 \\
Future 10S- & Imaging & 2.3 & 3.3 & 4.7 & 6.2 \\
$\quad$ BL & & & & & \\
\hline \hline
\end{tabular}

drift optics case. While SPEAR3 employs a number of wiggler insertion devices, the larger emission cone of a wiggler is not a well suited to pulse length selection by either the drift or imaging techniques illustrated in Fig. 2. Accordingly, the calculation results depicted in Fig. 3 assume wigglers are replaced by undulators with photon radiation divergence comparable to that of the in vacuum undulator on BL15. These beam lines could potentially be modified to use modern undulators in the future.

The photon beam distributions have also been calculated for the case with imaging optics between the source points and the slits. As pointed out earlier, the short pulse performance with imaging optics tends to be better at beam lines which are not ideal for drift optics. For example, the 10S straight section has a small $y^{\prime}-z$ slope because it is exactly half way across the ring from the crab cavities and thus does not have good short pulse performance with the drift optics. However, it is about the best location to achieve good short pulse performance with the imaging optics. A dedicated short pulse beam line with an in-vacuum undulator located in the 10S straight section and an imaging system for short pulse selection would have an excellent short-pulse performance, despite the degradation effects of the mirrors.

Table II shows the short pulse performance for two existing beam lines and the future 10S beamline. The short pulse duration is given for four levels of short pulse flux percentage of the total bunch. The flux percentage outside of the parenthesis is without bunch lengthening. When the bunch charge increases, the camshaft bunch lengthens. Although the tilt slope does not change, the charge density of the selected pulse will decrease, hence the selected flux percentage will decrease accordingly. Bunch lengthening for two SPEAR3 lattices have been measured for various bunch currents [15]. The momentum compaction factor for the 7-nm 2FCC lattice is between the two measured lattices. Based on the measurements, we estimate that the camshaft bunch will lengthen by $60 \%$ with a bunch current of $20 \mathrm{~mA}$ (corresponding to a bunch charge of $15.6 \mathrm{nC}$ ). The flux percentage in the parenthesis in the table includes bunch lengthening for a $20 \mathrm{~mA}$ camshaft bunch.

The minimum pulse duration reaches $3 \mathrm{ps}$ (fwhm). For a $20 \mathrm{~mA}$ camshaft bunch, the short pulse flux corresponds to radiation from an effective charge of $0.56 \mathrm{nC}$. When the slit is opened to select a pulse duration of $7 \mathrm{ps}$ (fwhm), the effective short pulse bunch charge is $2.0 \mathrm{nC}$. With a $20-\mathrm{mm}$ period, 170-period in vacuum undulator located at $10 \mathrm{~S}$, the photon flux reaches $0.9 \times 10^{12}$ photons/ sec within a $10^{-4}$ energy bandwidth for a 10-ps fwhm pulse. The flux would be higher if two camshaft bunches are stored.

\section{BEAM DYNAMICS ISSUES OF THE TILTED BUNCH}

In the 2FCC scheme, the stored beam is at the zero crossing of the crab cavity rf waveform. Because the stored beam is typically very short compared to the rf wavelength, it predominantly sees only the linear slope of the time dependent kicks. However, the injected beam is much longer than one wavelength and will thus see the full waveform. Touschek particles with large momentum deviations will also oscillate with large longitudinal amplitude and may see a significant fraction of the full waveform. The crab cavity can affect the injection efficiency and the Touschek lifetime of the tilted bunch. In this section we investigate these issues.

\section{A. Injection into the tilted bunch}

The rms bunch length of the injected beam from the SSRL Booster is $\sigma_{z \text {,inj }}=42 \mathrm{~mm}$. The $5 \sigma$ full length covers more than two full wavelengths for the $3 \mathrm{GHz}$ crab cavity. Portions of the injected beam see the full deflecting kick of $\theta_{\max }=\frac{e V_{d}}{E}$. The large vertical kick would drive the affected portions of the injected beam to large oscillation amplitudes and cause beam loss at injection.

One potential solution is to turn off the crab cavities shortly before injection, wait until injection is completed, and turn them back on. However, presently SPEAR3 employs frequent top-off fills with an interval of 5 minutes. Through an evaluation of the complication to the rf system, it was determined that it is not feasible to switch on and off the crab cavities with such frequency. Therefore, injection into the tilted bunch has to be compatible with the crab cavities at their operation strengths.

The oscillation amplitude of a kicked beam in a linear lattice can be calculated straightforwardly. Because the kick is stationary at a given longitudinal phase and the synchrotron motion is slow, the particles see the same kick for many turns before moving to a significantly different longitudinal phase. Therefore, the injected beam effectively has a different closed orbit from the stored beam. An injected particle that comes in on the mid-plane will start to oscillate around the distorted closed orbit with an amplitude equal to its initial distance from the closed orbit. Therefore, 
the maximum offset from the mid-plane will be twice that of the oscillation amplitude. Since the oscillation amplitude is limited by the vertical physical apertures, the maximum deflecting voltages by the crab cavities can be estimated through

$$
\frac{\sqrt{\beta_{1} \beta_{2}}}{2 \sin \pi \nu_{y}} \theta_{\max } \times 2=y_{\min }
$$

where $y_{\min }$ and $\beta_{1}$ are the half aperture and the vertical beta function at the location of the limiting aperture, respectively, and the factor of two on the left-hand side is to account for the closed orbit offset and the oscillation amplitude as discussed in the above.

For SPEAR3, the limiting half-aperture is $y_{\min }=$ $3.5 \mathrm{~mm}$ at $\beta_{1}=5.0 \mathrm{~m}$. Using other parameters found in Table I, the maximum allowed kick is calculated to be $\theta_{\max }=0.84 \mathrm{mrad}$. The corresponding maximum deflecting voltage on the 6th harmonic crab cavity is $V_{1, \max }=1.25 \mathrm{MV}$, with the two crab systems roughly contributing equal shares of kicks. Although this is higher than the nominal deflecting voltage of $V_{1}=1 \mathrm{MV}$, there could still be an impact to the injection efficiency as the injected beam has a finite vertical size and the beam could be driven to higher vertical amplitude through linear and nonlinear coupling.

The impact of the crab cavities to the dynamic aperture can be evaluated with particle tracking. Figure 4 shows a comparison of the dynamic aperture for the nominal crab cavity lattice with $V_{1}=1 \mathrm{MV}$ and without the crab cavities $\left(V_{1}=0\right)$. The solid lines represent the average dynamic aperture of 15 random seeds of linear optics and coupling errors for each case. The dashed lines represent the rms spread of the 15 seeds. A small vertical physical aperture of $3.5 \mathrm{~mm}$ at the septum location is included in the tracking simulation to determine the dynamic aperture. The sextupole strengths have been set to optimize the dynamic aperture of the lattice in a model without the crab cavities. The optimization was done with the multiobjective particle swarm optimization (MOPSO) algorithm with a setup similar to what was used in Ref. [16]. Figure 4 clearly shows a significant reduction of dynamic aperture by the crab cavities. Fortunately, even with the reduced dynamic aperture for the tilted bunch, the lattice is still workable with a new septum magnet that will soon replace the existing one. The new septum magnet will reduce the dynamic aperture requirement by using a much thinner wall between the stored beam and the injected beam.

In the dynamic aperture simulation, all particles are launched without energy or longitudinal phase errors. The tracked particles do not receive large vertical kicks from the crab cavities due to their initial phase offsets. However, a large fraction of the injected particles will see the maximum kick by the crab cavities due to the long injected beam bunch length. To fully include the impact of the crab

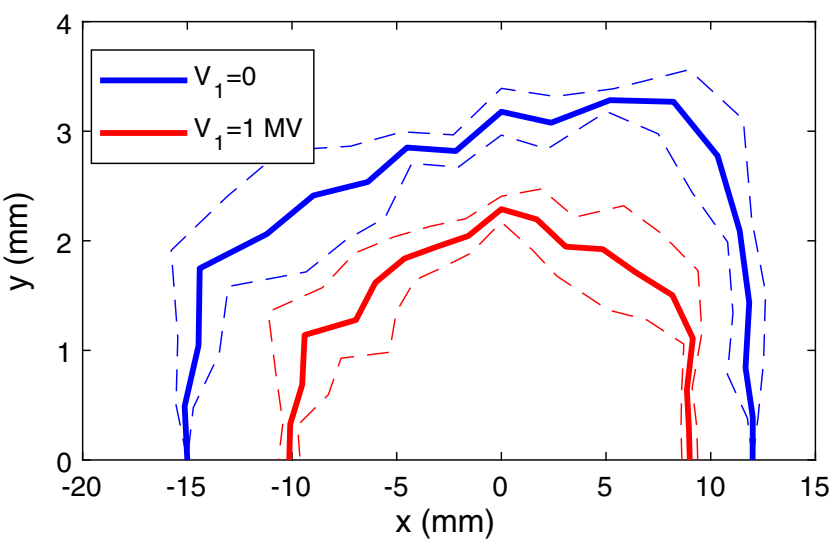

FIG. 4. Comparison of the storage ring dynamic aperture at the septum with two-frequency crab cavities $\left(V_{1}=1 \mathrm{MV}\right)$ and without crab cavities. A vertical physical aperture of $3.5 \mathrm{~mm}$ is in the lattice model. The solid lines are average values of 15 random seeds of linear optics and coupling errors in each case. The dashed lines represent the "error bars" calculated with the standard deviations. Dynamic aperture is evaluated by tracking particles for 5000 turns with radiation damping turned on.

cavities to the injected beam, we have simulated the injection process by tracking a realistic distribution of the injected beam.

An analysis has shown that the injected beam reaches an equilibrium distribution in the Booster synchrotron [17]. The parameters of the distribution are determined by the Booster lattice. The horizontal emittance is $\epsilon_{x}=170 \mathrm{~nm}$, the bunch length is $\sigma_{t}=140 \mathrm{ps}$, and the momentum spread is $\sigma_{\delta}=0.001$. The vertical emittance results from the linear coupling in the Booster and is estimated to be $\epsilon_{y}=17 \mathrm{~nm}$. The horizontal and vertical emittances have been verified with quadrupole scan measurements in the Booster-toSPEAR (BTS) transport line. The Courant-Snyder parameters and dispersion functions at the end of BTS are $\beta_{x}=\beta_{y}=5 \mathrm{~m}, \alpha_{x}=\alpha_{y}=0$, and $D_{x, y}=D_{x, y}^{\prime}=0$ for the design BTS optics. The values of these parameters in the real machine may differ from the design due to various errors, which could cause a reduction of injection efficiency. Beam-based correction and optimization methods may be used compensate the errors. The injected beam is launched with an initial horizontal offset of $x_{0}=-7.5 \mathrm{~mm}$, which leaves a space of $6 \sigma$ for the stored beam (one side), $3 \sigma$ for the injected beam (one side), a $2.5 \mathrm{~mm}$ septum wall, and a $0.6 \mathrm{~mm}$ tolerance for errors. The lattice model includes radiation damping, quantum excitation, and a vertical physical aperture of $3.5 \mathrm{~mm}$. The particles are tracked for 10000 turns in the ring.

The particle loss ratio as a function of the number of turns after injection is shown in the top plot of Fig. 5 for a few deflecting voltage levels. For the nominal case, with $V_{1}=1 \mathrm{MV}$, the total injection loss ratio is $5.5 \%$. The injection loss ratio of this level would be acceptable for operation, especially since this loss rate applies only to the 

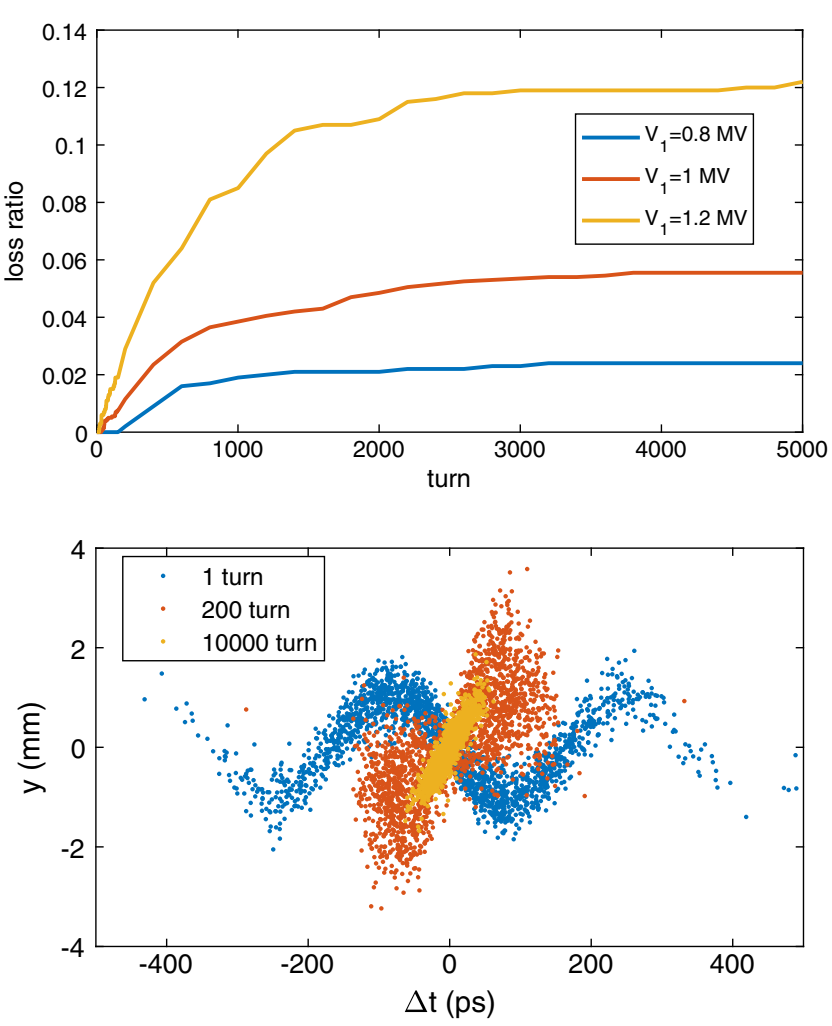

FIG. 5. Top: injection loss ratio vs number of turns for crab cavity voltages $V_{1}=0.8,1$, and $1.2 \mathrm{MV}$. Bottom: the injection beam distribution in $y-\Delta t$ coordinates for the $V_{1}=1 \mathrm{MV}$ case after the first turn, 200 turns, and 10,000 turns. The vertical acceptance corresponds to a $\pm 3.5 \mathrm{~mm}$ vertical aperture at the location.

tilted bunch. The particle distributions in the $y-\Delta t$ plane at the injection point after the first turn, 200 turns, and 10,000 turns are shown in the bottom plot of Fig. 5. The first turn distribution clearly shows the deflecting waveform of the crab cavities.

\section{B. Touschek lifetime for the tilted bunch}

The beam lifetime of a low emittance, high current electron bunch is dominated by the Touschek scattering loss. Touschek lifetime depends strongly on the momentum aperture. Because the Touschek particles oscillate in the longitudinal plane following the initial energy gain or loss, they could acquire a large longitudinal phase offset. Particles with large longitudinal phase shifts will receive significant vertical kicks from the crab cavities, which could lead to particle loss. In other words, the presence of the crab cavities would reduce the momentum aperture. This effect can be studied by evaluating the momentum aperture with particle tracking simulations.

In the simulation particles are launched from locations throughout the ring and tracked for 2000 turns. At each location, the initial momentum deviation ranges from $-4 \%$ to $4 \%$, with a step size of $0.1 \%$. The momentum aperture is determined by the initial momentum deviation of the

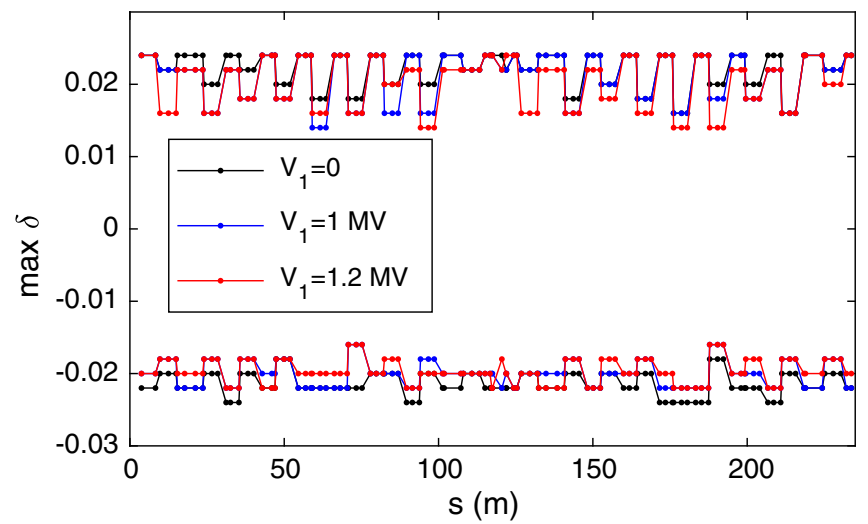

FIG. 6. The local momentum aperture determined from particle tracking simulation with crab cavities turned off $\left(V_{1}=0\right)$, or with deflecting voltage at $V_{1}=1 \mathrm{MV}$ or $V_{1}=1.2 \mathrm{MV}$.

surviving particles, after excluding any surviving particles that have larger initial momentum deviations than a lost particle. Figure 6 shows the local momentum aperture in the ring with crab cavities turned off and at two deflecting voltage levels, $V_{1}=1$ and $1.2 \mathrm{MV}$. The crab cavities clearly impact the local momentum aperture. However, the reduction of momentum aperture is not significant. The calculated Touschek lifetime for the tilted bunch with a $20-\mathrm{mA}$ bunch current is about $2 \mathrm{hrs}$. The beam loss from the tilted bunch in the 5-min interval between top-off fills is $0.8 \mathrm{~mA}$. Beam loss at this level is acceptable. Table III lists beam lifetime related parameters for three levels of deflecting voltages. Bunch lengthening by $60 \%$ for the $20 \mathrm{~mA}$ camshaft bunch has been included in the calculation. It is worth noting that the Touschek lifetime is longer for higher deflecting voltages. This is because the vertical slice emittance is increased for higher deflecting voltages, which reduces the Touschek scattering rate and in turn the Touschek beam loss.

\section{Effects of rf multipoles}

An ideal crab cavity is a rf dipole which should give a constant kick across the aperture of the cavity at any rf phase. However, an actual crab cavity would have field strength variations in the transverse directions. These variations correspond to higher order rf multipoles. Because of the symmetry in the cavity structure, the leading rf multipole in a crab cavity is a rf skew sextupole. The rf

TABLE III. Touschek lifetime, $\tau_{T}$, for the tilted bunch with a $20 \mathrm{~mA}$ bunch current and the deflecting voltage of the first frequency, $V_{1}$, at three levels. Also listed are the vertical slice emittance, $\epsilon_{y}$ and beam loss over the 5-min interval between fills.

\begin{tabular}{lccc}
\hline \hline$V_{1}(\mathrm{MV})$ & $\epsilon_{y}(\mathrm{pm})$ & $\tau_{T}(\mathrm{hr})$ & $\Delta I_{5-\min }(\mathrm{mA})$ \\
\hline 0.8 & 54 & 1.73 & 0.94 \\
1 & 80 & 2.03 & 0.80 \\
1.2 & 120 & 2.30 & 0.71 \\
\hline \hline
\end{tabular}



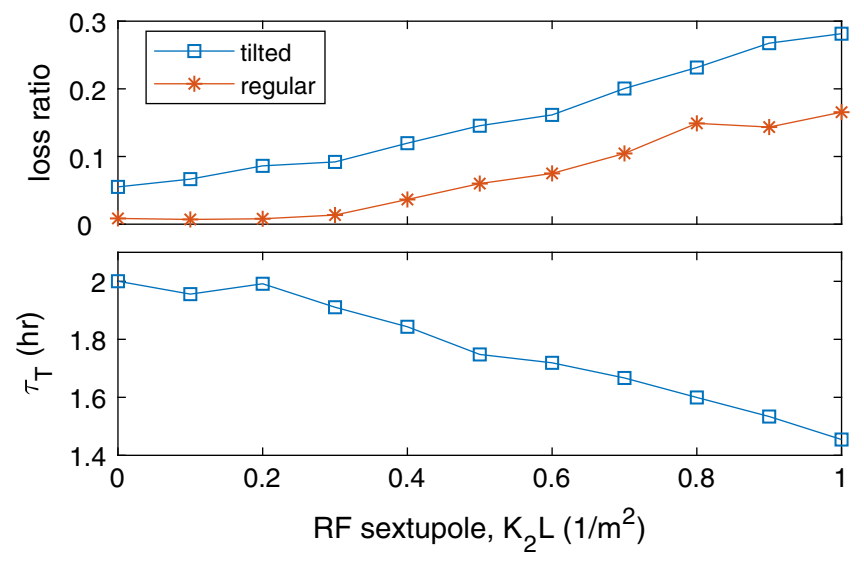

FIG. 7. The impact of rf skew sextupole to injection loss ratio (top) and camshaft Touschek lifetime (bottom). The deflecting voltage is $V_{1}=1 \mathrm{MV}$.

multipole could have an impact on the dynamic aperture and momentum aperture of the lattice. Simulation has been done to evaluate these effects.

In the simulation we insert one rf skew sextupole at the center of the crab cavity straight section. This is to represent the worst-case scenario when the rf multipoles in the crab cavities add up, instead of canceling each other. Figure 7 shows the injection loss ratio and the camshaft (at $20 \mathrm{~mA}$ ) Touschek lifetime. The rf skew sextupole increases the injection loss ratio for both the regular bunches and the camshaft bunch. It also decreases the Touschek lifetime. From these simulations we make the reasonable choice to specify that the total integrated rf skew sextupole strength from the crab cavities be below $0.2 \mathrm{~m}^{-2}$.

\section{SINGLE PARTICLE DYNAMICS IMPACT TO THE REGULAR BEAM}

Due to the cancellation of the deflecting slopes by the two frequencies, the impact of the crab cavities to the regular beam bunches should be negligible under the ideal conditions. However, the rf fields in the crab cavities will always have random noise, which translates to random kicks to the beam. The rf noise could increase the emittance of the regular beam and have a negative impact to the high brightness beam users. The regular beam consists of a long bunch train or a few shorter bunch trains with ion clearing gaps. The transient beam loading effects in the main rf due to the gaps will shift the arrival time of the bunches according to their positions in the bunch pattern. The phase errors cause imperfect cancellation which may also increase the vertical emittance. These two effects have been studied and the results are presented below.

\section{A. Effects of rf noise}

The rf noise in the crab cavities may be divided into three categories according to its frequency content. High frequency rf noise is defined as the components that have frequencies at the revolution frequency or higher. The high frequency noise can be modeled as white noise if we consider the kicks by the noise on consecutive beam revolutions are uncorrelated. In SPEAR3, the high frequency noise is at $1 \mathrm{MHz}$ or higher. Low frequency rf noise is the components with frequencies at about $1 \mathrm{kHz}$ or lower. The period of the low frequency noise is comparable to the radiation damping time. The low frequency components are also within the bandwidth that could be mitigated with feedbacks. The medium frequency noise is between the high and low frequency noise spectra, roughly from $1 \mathrm{kHz}$ to $1 \mathrm{MHz}$.

The emittance increase due to the random excitation of kicks by high frequency white noise has been studied in Ref. [10]. The formula is reproduced below

$\Delta \epsilon_{y} \approx \frac{1}{2}\left(\frac{e V_{1}}{E_{0}}\right)^{2} \frac{\tau_{y}}{T_{0}} \beta_{2} e^{-a_{1}^{2}}\left[\sinh \left(a_{1}^{2}\right) \sigma_{A}^{2}+\cosh \left(a_{1}^{2}\right) \sigma_{\phi}^{2}\right]$,

where $\tau_{y}$ is the vertical damping time, $T_{0}$ is the revolution time, $\beta_{2}$ is the vertical beta function at the crab cavity location, $a_{1}=\omega_{1} \sigma_{t}, \sigma_{t}$ is the bunch length in time, $\sigma_{A} \equiv$ $\sigma_{V} / V$ is the fractional amplitude noise sigma (i.e., rms value), and $\sigma_{\phi}$ is the rf phase noise sigma. The value calculated with Eq. (17) is lower than the valued calculated with the formula given in previous studies [10] by a factor of 4 because we assume the noise in the two frequencies are not correlated. If we require the contributions from the amplitude noise and the phase noise to be equal and the induced emittance increase to be below $10 \mathrm{pm}$, the relative amplitude and phase noise sigmas should satisfy $\sigma_{A}<2.5 \times 10^{-4}$ and $\sigma_{\phi}<0.9 \times 10^{-4} \mathrm{rad}$, respectively.

Particle tracking simulation was done to study the emittance increase due to high frequency rf noise. The amplitude and phase errors are modeled as random errors with a Gaussian white noise distribution. Figure 8 shows the projected emittance of the regular bunch as a function of the amplitude and phase noise levels. The projected emittance includes the contribution from linear coupling, about $4 \mathrm{pm}$ for a coupling ratio of $0.06 \%$ for the $7-\mathrm{nm}$ lattice. The simulation results indicate the requirement is a little more relaxed than the values given by Eq. (17). It would be acceptable to have noise levels (white noise)

$$
\sigma_{A}<4.0 \times 10^{-4} \text { and } \sigma_{\phi}<2.0 \times 10^{-4} \mathrm{rad} .
$$

The total vertical emittance for the untilted beam would be below $15 \mathrm{pm}$. The rf phase stability requirement may be challenging, slightly exceeding current demonstrated performance on existing pulsed systems. Future work is needed to evaluate the phase stability performance of continuous-wave (CW) operation of S-band cavities and possibly to develop mitigation. 


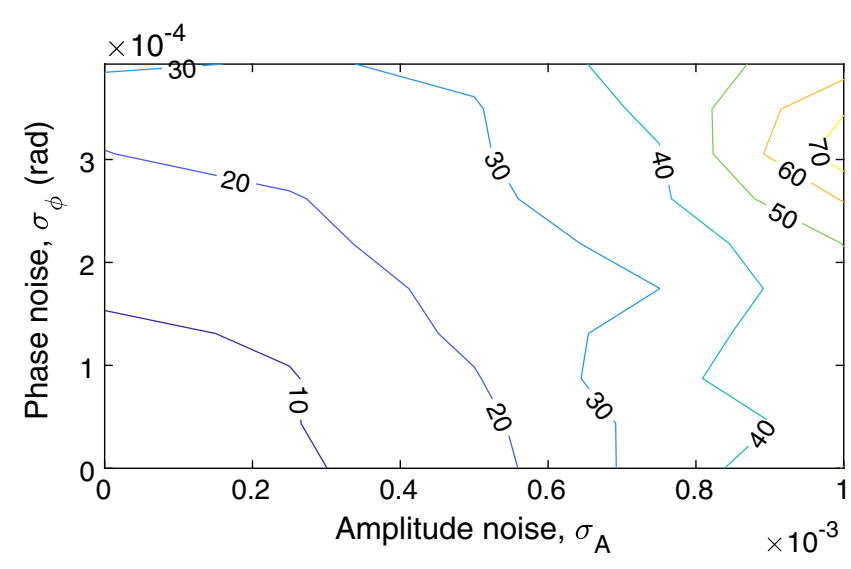

FIG. 8. Projected emittance ( $\mathrm{pm})$ of the regular bunches due to high frequency rf noise (modeled as white noise) in crab cavities (with $V_{1}=1 \mathrm{MV}$ ) vs levels of rf phase and amplitude noise. Emittance due to linear coupling, with a coupling ratio of $0.06 \%$, and the effect of a 10-ps phase shift (see next subsection) are included.

It is worth noting that high frequency phase modulations are not as detrimental as white noise errors. In a tracking simulation with a lattice without $x-y$ coupling, the crab cavity rf phase of the second frequency is modulated by a $1-\mathrm{MHz}$ oscillation with an amplitude of $9 \times 10^{-4} \mathrm{rad}$. The projected emittance of the bunch is about $0.05 \mathrm{pm}$, mostly coming from the residual curvature of the cancelled crab cavity waveforms. The effective emittance due to the jitters of the bunch centroid is $0.2 \mathrm{pm}$. When the modulation amplitude is increased to $9 \times 10^{-3} \mathrm{rad}$, the effective emittance due to the jitters of the bunch centroid is $20 \mathrm{pm}$.

The low frequency rf noise has several effects. The phase errors cause vertical kicks. The average kick (over the duration of the bunch) will drive coherent vertical orbit motion. Because of the low frequency, the kicks persist for enough turns such that the net effect is a varying closed orbit, rather than turn-to-turn betatron oscillation. The vertical kick for a phase error $\Delta \phi$ is $\Delta y^{\prime}=\frac{e V_{d}}{E} \Delta \phi$. For phase noise of $\sigma_{\phi}=1.0 \mathrm{mrad}$, the corresponding vertical kick is $\sigma_{y^{\prime}}=0.33 \mu \mathrm{rad}$, which causes an orbit shift of $1 \mu \mathrm{m}$ at the insertion devices for the SPEAR3 lattice. The phase errors also change the deflecting slope, which represents the variation of the vertical kick across the bunch length. But this slope change is a second order effect and is negligible.

The rf amplitude noise causes a mismatch between the deflecting slopes of the two frequencies. This mismatch corresponds to a residual deflecting slope. Because a slope error lasts for many turns, on the order the damping time, its net effect is to change the equilibrium distribution. The rms residual slope can be estimated from the relative voltage error with

$$
\sigma_{\epsilon}=\frac{\sqrt{2}}{2} \sigma_{A} \epsilon
$$

where $\epsilon$ is the combined deflecting slope from both frequencies. The vertical emittance for the regular beam due to the amplitude noise can then be obtained by scaling the vertical emittance of the tilted bunch with the factor $\frac{\sigma_{A}^{2}}{2}$. The projected emittance for the $V_{1}=1 \mathrm{MV}$ case is a constant throughout the ring at $1.6 \mathrm{~nm}$. For beamline users the effective emittance, $\sigma_{y} \sigma_{y^{\prime}}$, is probably more relevant because the beamline optics is not matched to the distorted $y-y^{\prime}$ phase space ellipse. The effective emittance varies from $2 \mathrm{~nm}$ to $20 \mathrm{~nm}$ for the existing and potential future ID locations in SPEAR3. The effective emittance growth at $\sigma_{A}=0.005$ is only up to $0.5 \mathrm{pm}$.

The effect of noise at intermediate frequencies between $1 \mathrm{kHz}$ and $1 \mathrm{MHz}$ would exhibit a behavior between that of the high and low frequency noise discussed in the above. It is expected that toward the high frequency end, the noise effect is more like the random excitation of the white noise. Toward the low frequency end, the noise behavior is more like the low frequency component, which drives slow motion on the closed orbit due to time-correlated kicks. While the noise requirement is more stringent at the high frequency end, it is also easier to achieve as there are typically fewer noise sources at $100 \mathrm{kHz}$ or higher. At the low frequency end, the noise requirement is more relaxed. Fast feedback in the low level rf systems would help mitigate the low frequency if noise.

\section{B. Effects of transient beam loading}

With ion clearing gaps in the bunch train, the fields in the main rf cavities vary with time within each $\mathrm{rf}$ period as the beam induced components of the fields (beam loading) change. The field variation changes the effective rf voltage for each bunch, which in turn cause the bunches to shift in phase in order to balance the radiation energy loss with the energy gain from the cavities. The phase changes will affect the deflecting slope cancellation of the two frequencies for the regular bunches and can have an impact on the user experiments. To assess this effect, measurements were carried out on the SPEAR3 ring to determine the arrival time variation across the bunch train.

Measurements were done with the bunch-by-bunch feedback system [18] for a few different fill patterns. Figure 9 shows the measured arrival time distribution over the bunch positions for two different patterns. One is a pattern with a long bunch train and the other has four shorter bunch trains and a $20 \mathrm{~mA}$ camshaft bunch. In both cases, the beam current was at $500 \mathrm{~mA}$. The long bunch train pattern has a total of 280 bunches. The second pattern is the camshaft pattern to be used for the $2 \mathrm{FCC}$ scheme. The mini gaps between the short bunch trains are 14 buckets wide. The large gap that houses the camshaft bunch in the middle is 59 buckets wide. Since there is a bigger gap for the first pattern, the arrival time difference is also bigger, at about $20 \mathrm{ps}$ from bunch train head to tail. 


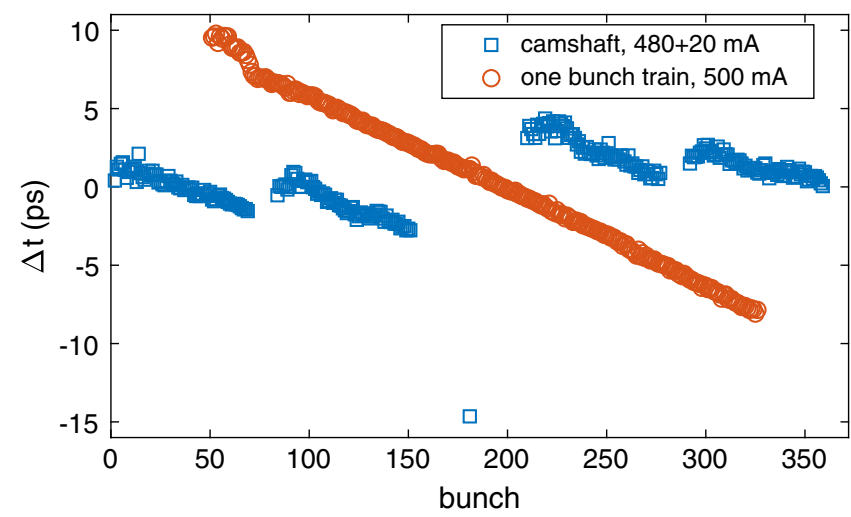

FIG. 9. Measured bunch arrival time distribution over the bunch train for two fill patterns in SPEAR3 with $500 \mathrm{~mA}$ total current and about 280 bunches.

The arrival time difference for the regular bunches in the camshaft pattern is up to $7 \mathrm{ps}$. The camshaft bunch itself is shifted by -15 ps from the other bunches.

Since the phase of the crab cavities will be adjusted to minimize the overall impact on the bunch train(s), the worst-case arrival time error for any bunch is one half of the maximum difference among the bunch train, i.e, $10 \mathrm{ps}$ for the case with one long bunch train, and 3.5 ps for the camshaft pattern.

When a bunch arrives at the crab cavities with a time shift, $\Delta t$, it will receive a net vertical kick

$$
\Delta y^{\prime}=\frac{e V_{1}}{E_{0}} \sin \left(\omega_{1} \Delta t\right)-\frac{e V_{2}}{E_{0}} \sin \left(\omega_{2} \Delta t\right) \approx 0,
$$

where the approximate cancellation is based on the fact that the two deflecting voltages are matched roughly according to $\omega_{1} V_{1}=\omega_{2} V_{2}$, with a small correction (about $1 \%$ for the SPEAR3 case) due to the intentional over-compensation proposed in Ref. [10].

The change of the deflecting slope is

$$
\begin{aligned}
\frac{d \Delta y^{\prime}}{d z} & =\frac{e k_{1} V_{1}}{E_{0}} \cos \left(\omega_{1} \Delta t\right)-\frac{e k_{2} V_{2}}{E_{0}} \cos \left(\omega_{2} \Delta t\right) \\
& \approx \frac{\epsilon}{4}\left(\omega_{2}^{2}-\omega_{1}^{2}\right) \Delta t^{2},
\end{aligned}
$$

where $\epsilon$ is the deflecting slope for the tilted bunch. For a 10-ps phase shift, the deflecting slope for the regular beam is only $0.0014 \epsilon$. The change of the effective emittance due to such a small slope is negligible.

In reality, because of the curvature of the sinusoidal waveform, the finite bunch length, and the intentional overcompensation in slopes, the vertical emittance may be slightly different. Simulation was performed to evaluate the emittance growth due to the gap transient induced phase shift. Figure 10 shows the equilibrium effective emittance (at a location with $20 \mathrm{~nm}$ effective emittance for the tilted bunch) for three levels of phase shifts, $0,10 \mathrm{ps}$, and $20 \mathrm{ps}$.

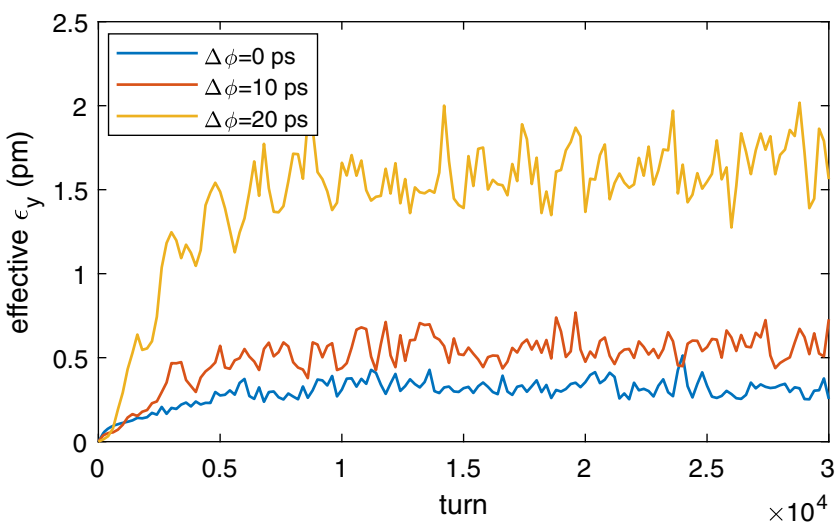

FIG. 10. Growth of effective emittance for the regular beam due to bunch arrival time shifts. There is no $x-y$ linear coupling in the lattice model.

The corresponding equilibrium effective emittances are $0.3,0.6$, and $1.6 \mathrm{pm}$, respectively. The corresponding equilibrium eigenemittances (not shown) are 0.05, 0.07, and $0.13 \mathrm{pm}$, respectively. The gap transient induced phase shift should not be a problem for the SPEAR3 crab cavity project.

\section{EVALUATION OF COLLECTIVE EFFECTS}

The addition of crab cavities to the storage ring could cause operational issues through collective effects of the beams. The resonant modes in the crab cavities can drive coupled bunch instabilities (CBI). The beam stability requirements put a limit on the impedances of the resonant modes in the cavities. The tilted bunch needs to host a high charge in order to produce high flux short pulses. On the other hand, single bunch instabilities, such as the microbunching instability and the transverse mode coupling instability (TMCI), limit the total charge in a bunch if it is to stay below the instability thresholds. In this section we assess the impact of the collective effects on the SPEAR3 2FCC scheme.

\section{A. Coupled bunch instabilities}

Because the bunch length is very short compared to the bunch spacing, typically in an electron storage ring the coupled bunch instabilities are limited to dipole mode oscillations. The analysis of such instabilities can be done by considering each bunch as a macroparticle. Analytic formulas for the instability threshold can be found in Ref. [19,20].

A longitudinal CBI can be driven by the monopole modes in the crab cavities, if the resonant frequency of a mode overlaps with the synchrotron sidebands of the beam revolution harmonics. For a uniform fill, the instability threshold can be calculated from the synchrotron tune, beam energy, momentum compaction factor, the total current, and the resonant frequency. For the SPEAR3 
7-nm lattice with a total current of $500 \mathrm{~mA}$, it is found that the threshold impedance is $Z_{l}^{\mathrm{th}}=8.3 \mathrm{k} \Omega$ for a mode at $3 \mathrm{GHz}$. Radiation damping is assumed as the only damping mechanism.

Overlapping of frequencies of dipole modes in the crab cavities with the betatron sidebands can drive transverse CBIs. The thresholds for uniform fills can be calculated from the beam energy, betatron tune, damping time, and the total beam current. SPEAR3 is equipped with a bunch-bybunch feedback system [18], which can mitigate transverse CBIs. The design damping time of the $\mathrm{BxB}$ system is $0.2 \mathrm{~ms}$. In a conservative estimate, we use a damping time of $1 \mathrm{~ms}$ in the threshold calculation. The resulting transverse impedance thresholds are $Z_{x}^{\text {th }}=4.7 \mathrm{M} \Omega / \mathrm{m}$ for the horizontal plane and $Z_{y}^{\text {th }}=1.9 \mathrm{M} \Omega / \mathrm{m}$ for the vertical plane.

\section{B. Single bunch effects}

When the bunch charge is increased in SPEAR3, the bunch will lengthen due to the inductive longitudinal impedance. Bunch lengthening in SPEAR3 has been studied in Ref. [15]. A longitudinal broadband impedance model has been derived from the bunch profile measurements under various rf voltage and lattice configurations. The longitudinal impedance is estimated to be $\frac{Z_{\|}}{n}=0.2 \Omega$.

With more charge added to a bunch, the beam can become unstable in the longitudinal plane, resulting in an increase of the momentum spread and bursts of microbunching in the longitudinal profile. Using the Boussard criterion [20,21] and the above longitudinal impedance model, the microbunching instability (MBI) threshold for the bunch charge SPEAR3 is calculated to be $12 \mathrm{~mA}$. Experimental measurements of the SPEAR3 microbunching instability were conducted by measuring the horizontal beam size with the pinhole camera. Because the photon source point of the pinhole camera is a dispersive location, the measured horizontal beam size depends on the momentum spread. Figure 11 shows the difference of the measured horizontal beam size between the single bunch mode and the 280-bunch mode for the same total current. Horizontal beam size of the latter is subtracted to remove the effects of camera exposure time and gain. The abrupt increase of horizontal beam size at $I_{b}=40 \mathrm{~mA}$ should signify the onset of microbunching instability. The measured MBI threshold is much higher than the calculated value, which could either be due to an inaccurate impedance model, or some damping mechanisms. It seems reasonable to operate with a $20 \mathrm{~mA}$ camshaft bunch for the $2 \mathrm{FCC}$ crab cavity scheme.

With a high charge in a single bunch, the beam can also become unstable in the transverse planes. The TMCI instability limits the total bunch current $[19,20]$. When the vertical chromaticity is at the nominal value of $C_{y}=2$, the single bunch current limit is $22 \mathrm{~mA}$, at which point any

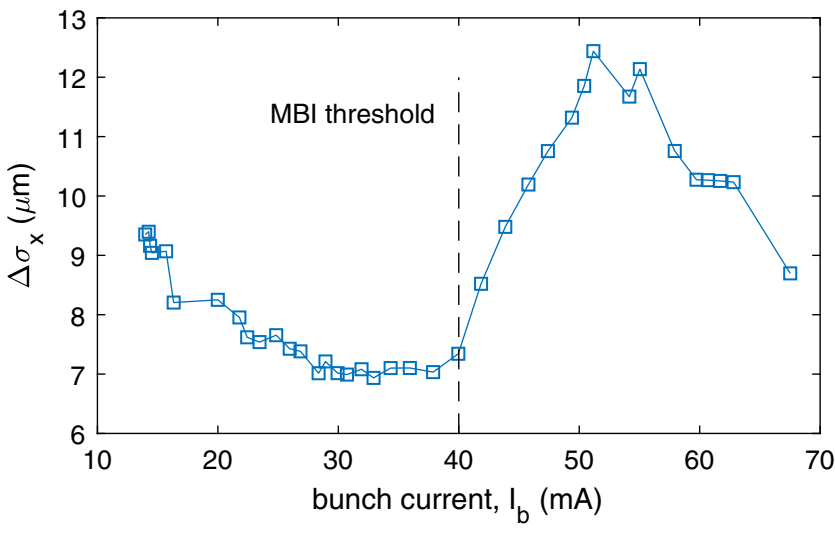

FIG. 11. Difference between the horizontal beam size measured in single bunch mode and multi-bunch mode for the same total current. The multi-bunch beam size is subtracted to remove effects of camera exposure time and gain. With vertical chromaticity at $C_{y}=5$, up to $70 \mathrm{~mA}$ in a single bunch has been stored in SPEAR3.

attempt to inject more charge causes step losses. However, when the vertical chromaticity is increased to $C_{y}=5$, the single bunch current limit is above $70 \mathrm{~mA}$, as was shown in Fig. 11.

The TMCI is determined by the short range transverse wakefields. The transverse impedance in the SPEAR3 ring has been characterized in experiments through a series of tune shifts vs bunch current measurements, from which a broad-band resonator model was obtained [22]. Combining the broad-band resonator impedance model and the calculated resistive wall impedance, the total kick factor of the ring is calculated to be $k_{y}=2000 \mathrm{~V} /(\mathrm{pC} \cdot \mathrm{m})$ for the vertical plane with a bunch length of $\sigma_{t}=30 \mathrm{ps}$ (including bunch lengthening). The crab cavities would contribute additional broadband impedance due to the transitions in the cavity structures. To avoid a significant reduction of the TMCI threshold, we specify that the total kick factor from the crab cavities cannot exceed $k_{y}=1500 \mathrm{~V} /(\mathrm{pC} \cdot \mathrm{m})$.

\section{OTHER PRACTICAL CONSIDERATIONS}

\section{A. Crab cavity options}

To achieve the desired short pulse performance, a certain total deflecting slope is required. To provide such a slope, the product of the crab cavity frequency and the deflecting voltage is fixed. The choice of crab cavity frequencies can be varied, along with the deflecting voltages. We chose the first crab cavity frequency to be a $n_{1}=6$ harmonic of the main $\mathrm{rf}$ frequency. It would seem desirable to choose a higher harmonic, such as $n_{1}=9$, as it will reduce the required deflecting voltage and may also occupy less space in the ring. However, a high frequency poses challenges in several aspects as it typically requires a smaller aperture. The physical aperture requirement for SPEAR3 is set to $36 \mathrm{~mm}$ horizontal and $12 \mathrm{~mm}$ vertical. A warm crab cavity 
has been designed for the SPEAR3 2FCC scheme [23], for which (with $n_{1}=6$ ) the full aperture is already at $40 \mathrm{~mm}$. Smaller aperture also leads to higher short range wakefields, which could exceed the specification. The warm crab cavity design has a total vertical kick factor of $k_{y}=1300 \mathrm{~V} /(\mathrm{pC} \cdot \mathrm{m})[23]$.

Choosing a lower frequency will make it easier to meet the short range wakefield requirement. However, a high deflecting voltage is needed, which would require more space in the ring. More importantly, as shown in Eq. (16), the maximum allowed vertical kick by the crab cavities is given by the fixed vertical physical aperture. With $n_{1}=6$, the maximum deflecting voltage is $1.25 \mathrm{MV}$, and at $V_{1}=1 \mathrm{MV}$, there is already a significant reduction in dynamic aperture. A higher deflecting voltage (and hence the maximum vertical kick) will cause high injection loss.

The crab cavities need to work in continuous-wave (CW) mode with high current beams. Superconducting crab cavities would be suitable for avoiding problems from beam-induced heat load. However, the warm crab cavity design can also control the heat power to an acceptable level [23]. The 13-cell warm crab cavity also meets our other specifications, such as deflecting voltage, longitudinal footprint, maximum impedance for unwanted resonance modes, beam aperture, short range wakefields, and the transverse uniformity of the deflecting field. The superconducting quasiwaveguide multicell deflecting cavity design developed for the APS-SPX project [24] could also be used, although the study in Ref. [23] showed that it would not meet the field uniformity requirement and more design optimization work is required.

\section{B. Separation of short pulses and high brightness beams}

User experiments that use the short pulses typically want to separate the high average current background from the short pulses. As the time separation between the camshaft bunch and the regular bunches is less than $100 \mathrm{~ns}$, it is difficult to block the high brightness beam with a mechanical chopper. Pseudo single-bunch operation [25], a method that creates transverse orbit separation between different bunches with a fast kicker, could be used.

In the 2FCC scheme the pseudo single bunch approach can be easily applied to achieve the separation between the camshaft bunch and the other bunches since the crab cavities are naturally fast vertical kickers. If the phases of the two crab cavity systems are shifted by 0.2 rad (for $n_{1}=6$ ) and $0.215 \mathrm{rad}$ (for $n_{2}=6.5$ ), respectively, the tilted bunch will receive a net vertical kick of $133.3 \mu \mathrm{rad}$. The vertical kicks apply to the camshaft every turn, which puts it on a closed orbit different from that of the regular bunches. The effects of the phase shifts to the regular bunches are the same as a 10-ps phase shift due to the gap

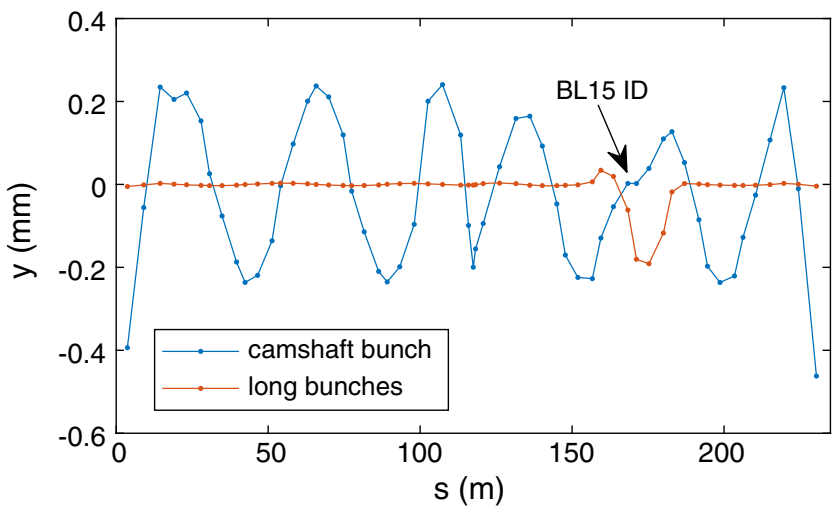

FIG. 12. Separated closed orbits for the camshaft bunch and the regular bunches when the crab cavity phases are shifted by $0.2 \mathrm{rad}$ and a local orbit bump is created at BL15 undulator.

transient beam loading effect, which was studied in the above and was found to not be a problem.

At the locations where there is a large $y^{\prime}-z$ tilt for the camshaft bunch, the vertical kicks will cause a large angular difference with the regular orbit. Conversely, at locations with a large $y-z$ tilt, there will be a large positional difference between the two orbits. In both cases, the orbit separation is ideal for the separation of the two photon beams. The angle and position for the camshaft bunch at the photon beam source point are shifted by the vertical kicks, which affect the beamline optics. This can be corrected with a local orbit bump using the corrector magnets. Figure 12 shows the two orbits when a local bump is created at the source point of BL15. The angle and position separation between the camshaft and regular bunches at BL15 is $\Delta y^{\prime}=-31.7 \mu \mathrm{rad}$ and $\Delta y=-159 \mu \mathrm{m}$, respectively. At the short pulse selection slit, assumed to be at $13.5 \mathrm{~m}$ downstream of the source point, the position separation is $587 \mu \mathrm{m}$, which is considerably larger than the spot size of the long bunches (with $\sigma_{y, \text { long }}=71 \mu \mathrm{m}$ ).

\section{SUMMARY}

We have studied beam physics topics related to the 2-frequency crab cavity scheme for short pulse production in storage rings [10]. With this scheme, high-flux short pulses can be simultaneously provided with the usual high current, high brightness beams. In the study the scheme is applied to the SPEAR3 storage ring. Using two crab cavity systems, one at $2.858 \mathrm{GHz}$ with a total deflecting voltage of $1 \mathrm{MV}$, and the other at $3.096 \mathrm{GHz}$ and $0.93 \mathrm{MV}$, beams in half of the buckets will be tilted, while beams in alternate buckets are minimally impacted by the crab cavities. A 20$\mathrm{mA}$ camshaft bunch will be filled to a tilted bucket, while regular beams are filled to the un-affected buckets. Short pulses can be obtained from the radiation of the tilted camshaft bunch by using vertical slits to select a portion of the photon pulse. 
The prediction of short pulse performance was analyzed. Its dependence on relevant machine and beam parameters are presented. Multiparticle simulation was used to obtained the short pulse performance for the SPEAR3 case for various beamlines. The short pulse flux is equivalent to a $0.56 \mathrm{nC}$ bunch with a $3 \mathrm{ps}$ (fwhm) pulse duration. Higher flux can be achieved with longer pulse duration, for example, flux equivalent to $2 \mathrm{nC}$ for a $7 \mathrm{ps}$ (fwhm) duration. The $8-\mathrm{keV}$ photon flux can reach $0.9 \times 10^{12}$ photons/ sec within a $10^{-4}$ energy bandwidth for a 10-ps fwhm pulse from a new 10S beamline.

The crab cavities can impact the injection efficiency and the beam lifetime of the camshaft bunch. Both issues were studied with particle tracking simulation. While there is a significant reduction of dynamic aperture by the crab cavities due to their vertical kicks and a small vertical acceptance, injection efficiency $>90 \%$ is still feasible. The Touschek lifetime of the 20-mA camshaft bunch is $2 \mathrm{hrs}$, which is acceptable with 5-min frequent fills. Rf skew sextupole component in the crab cavities was found to have a large impact to the dynamic aperture. An integrated skew sextupole component of $0.2 \mathrm{~m}^{-2}$ was specified to avoid a loss of injection efficiency.

Rf noise in the crab cavities can increase the vertical emittance of the regular beam. This was studied with both analytical analysis and simulation. Transient beam loading caused by the ion clearing gaps can introduce arrival time shifts in the bunches, which could in turn affect the vertical emittance of the regular bunches. Experiments were done to measure the arrival time shifts, which were found to be less than 10 ps for the worst-case fill pattern. Analysis and simulation showed that this level of phase shift is not a problem.

Collective effects, including coupled bunched instabilities and single bunch instabilities, were studied. CBI thresholds were given for both the longitudinal and transverse planes. Experiments were performed to determine the microbunching instability threshold and the transverse mode coupling instability (TMCI) threshold.

Separation of the radiation from the camshaft bunch and the regular bunches can be done with the pseudo single bunch approach [25], using crab cavities as the fast kickers.

In this study we applied the 2FCC scheme to SPEAR3, a third generation storage ring. The scheme would also be applicable to the next generation of storage rings based on multi-bend achromat lattices (MBA). There will be additional challenges in these rings. For example, the single bunch currents limit may be lower due to increased impedances and small momentum compaction factors. The effects of bunch lengthening harmonic cavities need to be studied. For rings with on-axis injection, the single bunch current will be also limited by the bunch charge of the injector. The Touschek lifetime would be lower due to reduced emittances. However, there are also advantages in applying the $2 \mathrm{FCC}$ scheme to the low emittance MBA rings. For example, the small momentum compaction factor diminishes the crab cavity-induced vertical emittance [see Eq. (5)], which allows choosing a vertical tune closer to the integer. A lower vertical tune reduces the required deflecting voltages for a given tilting angle [Eqs. (2)-(3)] and shortens the bunch length for the tilted beam due to the longitudinal focusing of the crab cavities (see Ref. [12]). Therefore, the 2FCC short pulse performance and operation issues for an MBA-based ring need to be specifically studied, with the same principles set forth in Refs. [10,12] and the present paper.

\section{ACKNOWLEDGMENTS}

We thank Zenghai Li and Valery Dolgashev for many helpful discussions. Work was supported by the U.S. Department of Energy, Office of Science, Office of Basic Energy Sciences, under Contract No. DE-AC0276 SF00515.

[1] X. Huang, J. Safranek, J. Corbett, Y. Nosochkov, J. Sebek, and A. Terebilo, Low alpha mode for SPEAR3, in Proceedings of the 22nd Particle Accelerator Conference, PAC-2007, Albuquerque, NM (IEEE, New York, 2007), pp. 1308-1310.

[2] W. Guo, B. Yang, C.-X. Wang, K. Harkay, and M. Borland, Generating picosecond X-ray pulses in synchrotron light sources using dipole kickers, Phys. Rev. Accel. Beams 10, 020701 (2007).

[3] J. Safranek, Y. Yan et al., Resonant crabbing for short pulses in SPEAR3 (unpublished).

[4] X. Huang, J. Safranek, and K. Bane, Injecting short pulses into SPEAR3, Stanford Synchrotron Radiation Lightsource (SSRL), Report No. SSRL-AP-note 65, 2015.

[5] X. Huang, T. Rabedeau, and J. Safranek, Generation of picosecond electron bunches in storage rings, J. Synchrotron Rad. 21, 961 (2014).

[6] G. Wustefeld et al., Simultaneous long and short electron nunches in the BESSY-II Storage Ring, in Proceedings of the 2nd International Particle Accelerator Conference, San Sebastiáán, Spain (EPS-AG, Spain, 2011).

[7] Technical Design Study of the BESSY Variable Pulse Length Storage Ring (VSR), http://dx.doi.org/10.5442/ R0001, Helmholtz-Zentrum Berlin, June 2015.

[8] A. Zholents, P. Heimann, M. Zolotorev, and J. Byrd, Generation of subpicosecond X-ray pulses using RF orbit deflection, Nucl. Instrum. Methods Phys. Res., Sect. A 425, 385 (1999).

[9] Conceptual Design Report, APS Upgrade Project, Report No. APSU-1.1-PLN-002-02.1, Argonne National Laboratory, 2011.

[10] A. Zholents, A new possibility for production of subpicosecond $\mathrm{x}$-ray pulses using a time dependent radio frequency orbit deflection, Nucl. Instrum. Methods Phys. Res, Sect. A 798, 111 (2015).

[11] X. Huang, Y. Nosochkov, and J. Safranek, Emittance upgrade for the SPEAR3 storage ring, ICFA beam 
dynamics newsletter (2017), Vol. 71, pp. 266-280, http:// www.icfa-bd.org/Newsletter71.pdf.

[12] X. Huang, Coupled beam motion in a storage ring with crab cavities, Phys. Rev. Accel. Beams 19, 024001 (2016).

[13] A. Terebilo, Accelerator modeling with Matlab Accelerator Toolbox, in Proceedings of the 19th Particle Accelerator Conference, Chicago, IL, 2001 (IEEE, Piscataway, NJ, 2001), pp. 3203-3205, Chicago (2001).

[14] M. Borland, Advanced Photon Source LS-287, September 2000.

[15] J. Corbett, W. Cheng, A. S. Fisher, and X. Huang, Bunch length and impedance measurements at SPEAR3, in Proceedings of the 11th European Particle Accelerator Conference, Genoa, 2008 (EPS-AG, Genoa, Italy, 2008).

[16] X. Huang and J. Safranek, Nonlinear dynamics optimization with particle swarm and genetic algorithms for SPEAR3 emittance upgrade, Nucl. Instrum. Methods Phys. Res., Sect. A 757, 48 (2014).

[17] X. Huang, Emittance of the SSRL Booster, Report No. SSRL-AP-Note 030, 2011.

[18] K. Tian, J. Corbett, X. Huang, N. Kurita, D. Martin, J. Safranek, and J. Sebek, Commissioning of transverse bunch-by-bunch feedback at SPEAR3, Proceedings of the 10th International Particle Accelerator Conference,
IPAC2019, Melbourne, Australia (JACoW Publishing, Geneva, Switzerland, 2019).

[19] A. Chao, Physics of Collective Beam Instabilities in High Energy Accelerators (John Wiley \& Sons, New York, 1993).

[20] Handbook of Accelerator Physics and Engineering, edited by A. Chao and M. Tigner (World Scientific, Singapore, 1999).

[21] D. Boussard, CERN Report LabII/RF/Int./75-2, 1975.

[22] X. Huang and J. Sebek, Impedance measurement for the SPEAR3 storage ring, Proceedings of the 6th International Particle Accelerator Conference, IPAC2015, Richmond, VA, USA (JACoW, Geneva, Switzerland, 2015), pp. 694697.

[23] Z. Li et al., Normal conducting CW transverse crab cavity for producing short X-ray pulses in SPEAR3, Proceedings of IPAC2017, Copenhagen, Denmark (2017), pp. 2840 2843, http://accelconf.web.cern.ch/AccelConf/ipac2017/ papers/wepab115.pdf.

[24] A. Lunin, T. Khabiboulline, I. Gonin, V. Yakovlev, and A. Zholents, Proceedings of LINAC'14, Geneva, Switzerland (2014), pp. 966-968, http://accelconf.web.cern.ch/ AccelConf/LINAC2014/papers/thpp051.pdf.

[25] C. Sun, D. Robin, C. Steier, and G. Portmann, Characterization of pseudosingle bunch kick-and-cancel operational mode, Phys. Rev. Accel. Beams 18, 120702 (2015). 